\title{
An electronic compendium of extreme functions for the Gomory-Johnson infinite group problem
}

\author{
Matthias Köppe*, Yuan Zhou \\ Department of Mathematics, University of California, Davis
}

\begin{abstract}
In this note we announce the availability of an electronic compendium of extreme functions for Gomory-Johnson's infinite group problem. These functions serve as the strongest cut-generating functions for integer linear optimization problems. We also close several gaps in the literature.
\end{abstract}

Keywords: integer programming, cutting planes, infinite group relaxation, software 2010 MSC: $90 \mathrm{C} 10$

\section{Introduction}

The infinite group problem was introduced 42 years ago by Ralph Gomory and Ellis Johnson in their groundbreaking papers titled Some continuous functions related to corner polyhedra I, II [8, 9]. The technique, investigating strong relaxations of integer linear programs by convexity in a function space, has often been dismissed as "esoteric." Now we recognize the infinite group problem as a technique which was decades ahead of its time, and which may be the key to today's pressing need for stronger, multi-row cutting plane approaches.

In this note, however, we restrict ourselves to the singlerow (or, "one-dimensional") infinite group problem, which has attracted most of the attention in the past. It can be written as

$$
\begin{aligned}
& \sum_{r \in \mathbb{R}} r y(r) \equiv f \quad(\bmod 1) \\
& y: \mathbb{R} \rightarrow \mathbb{Z}_{+} \text {is a function of finite support, }
\end{aligned}
$$

where $f$ is a given element of $\mathbb{R} \backslash \mathbb{Z}$. We study the convex hull $R_{f}(\mathbb{R}, \mathbb{Z})$ of the set of all functions $y: \mathbb{R} \rightarrow \mathbb{Z}_{+}$satisfying the constraints in (1). The elements of the convex hull are understood as functions $y: \mathbb{R} \rightarrow \mathbb{R}_{+}$.

After a normalization, valid inequalities for the convex set $R_{f}(\mathbb{R}, \mathbb{Z})$ can be described using so-called valid functions $\pi: \mathbb{R} \rightarrow \mathbb{R}$ via

$$
\langle\pi, y\rangle:=\sum_{r \in \mathbb{R}} \pi(r) y(r) \geq 1 .
$$

In the finite-dimensional case, instead of merely valid inequalities, one is interested in stronger inequalities such

\footnotetext{
* Corresponding author

Email addresses: mkoeppe@math.ucdavis.edu (Matthias Köppe), yzh@math.ucdavis.edu (Yuan Zhou)

Preprint submitted to OR Letters
}

as tight valid inequalities and facet-defining inequalities. These rôles are taken in our infinite-dimensional setting by minimal functions and extreme functions. Minimal functions are those valid functions that are pointwise minimal; extreme functions are those that are not a proper convex combination of other valid functions.

By a theorem of Gomory and Johnson [8, minimal functions for $R_{f}(\mathbb{R}, \mathbb{Z})$ are classified: They are exactly the subadditive functions $\pi: \mathbb{R} \rightarrow \mathbb{R}_{+}$that are periodic modulo 1 and satisfy the symmetry condition $\pi(x)+\pi(f-x)=$ 1 for all $x \in \mathbb{R}$. A major goal of research is to obtain a classification, or at least improved understanding, of the extreme functions as well.

We refer the interested reader to the recent surveys [6, 13] and the forthcoming survey [4] for a more detailed exposition.

The main purpose of this note is to announce the availability of an Electronic Compendium of Extreme Functions 16, implemented in Python within the framework of the open-source computer algebra system Sage [15]. We hope that it will facilitate new research on the infinite group problem, in particular by enabling computational experiments, including those that would investigate the strength of these functions to generate the coefficients of cutting planes in a branch and cut algorithm. To our knowledge, such experiments have not been conducted systematically with all known families of extreme functions.

The survey 13 provided an excellent service by organizing the extreme functions known at that time; yet our knowledge has grown since then, and our Electronic Compendium provides for the first time convenient and up-todate access to the definitions of the functions, bringing light to those functions hidden in obscurity.

The extremality proof for a given class of piecewise minimal valid functions follows a standard pattern, which we illustrate with the proof of extremality for a class of 
functions that was described in the literature but whose extremality was unknown before (section 2).

This standard proof pattern can actually be fully automated for a given piecewise minimal valid function with rational data [3]; see also [4, section 7]. The Compendium is released as part of software [1] that implements this automated extremality test and thus enables computational experiments to find new extreme functions, to make conjectures, and to verify claims in the literature. As an example regarding the latter, we highlight the case of a family of piecewise linear functions with 3 slopes studied by Chen [5]. Chen's extremality proof is flawed, and using our software we can easily determine the non-extremality of these functions section 3.

All definitions of extreme functions in the Compendium are provided with unit tests using this extremality-testing algorithm, which help to ensure the correctness of the compendium as we continue to add newly discovered functions or generalize their constructions.

Some classes of piecewise linear minimal valid functions have historically been proved extreme using a different type of proof, either by the connection to a sequence of finite group problems, or a lemma regarding limits of sequences of extreme functions. We may now consider these proofs obsolete, as they can be replaced by proofs following the standard pattern. We illustrate this by an example in section 4

The only example known in the literature whose extremality proof does not follow the standard pattern and cannot be automated is a family of non-piecewise linear, measurable functions introduced by Basu et al. 2] as a counterexample to a conjecture by Gomory-Johnson. Basu et al.'s functions are uniform limits of certain 2-slope functions, which we identify as kf_n_step_mir functions. (Throughout this paper, we refer to an extreme function or a family of extreme functions by the name of the Sage function that constructs them; these names are shown in typewriter font.) The limit functions can be seen as absolutely continuous, measurable, non-piecewise linear generalizations of 2-slope functions. In section 5, we contribute a discussion of a limiting case of the same construction, in which one of the two slopes disappears, giving a continuous (but not absolutely continuous), measurable, nonpiecewise linear "1-slope function." It can be seen as a periodic, subadditive version of Cantor's devil function.

At the end of the article section 6) we explain how to use the Electronic Compendium and show an overview of the functions in it at the time of writing of this article Table 1.

\section{Extremality of Richard et al.'s $\mathrm{DPL}_{1}$-extreme function $r l m_{-} d p l 1 \_$extreme_3a}

Richard et al. 14 study families of piecewise linear minimal valid functions with a prescribed set of breakpoints. (They present these results within their theory of approximate lifting, where pseudo-periodic superadditive functions appear. However, these can be transformed, via [14, Proposition 18], to the standard setting of minimal valid functions for the infinite group problem, which are periodic and subadditive. We describe all functions after applying this transformation.) Specifically, $\mathrm{DPL}_{n}$ functions are possibly discontinuous piecewise linear functions with $2 n+1$ subintervals in $[0,1]$, whose graphs include the closed line segment from $\left(\begin{array}{l}0 \\ 0\end{array}\right)$ to $\left(\begin{array}{l}f \\ 1\end{array}\right)$. They are parametrized by a finite number of real values: the slopes of the subintervals (whose lengths are fixed in advance) and the values of the jumps at the (fixed) breakpoints. Subadditivity of these functions is then characterized by finitely many linear inequalities [4, subsection 3.4. The functions corresponding to the extreme points of the polytope $P \Theta_{n}$ are called "DPL ${ }_{n}$-extreme functions". These functions are candidates for, but not guaranteed to be extreme functions for the infinite group problem, because perturbations may exist that are not piecewise linear on the same set of breakpoints but rather on a refinement; see [4, subsection 8.2.

For small $n$, the extreme points of $P \Theta_{n}$ and thus the resulting $\mathrm{DPL}_{n}$-extreme functions can be listed. Richard et al. [14, Theorem 28] accomplish this for $n=1$, giving piecewise formulas for the extreme points in terms of the single remaining breakpoint parameter $f$. For any value of $f$, there are three extreme points. Richard et al. 14 note that the first one corresponds to the gmic function and the second one recovers functions proved extreme in [7, namely, drlm_3_slope_limit(f) for $0<f<\frac{1}{2}$ and drlm_2_slope_limit (f, 1,1$)$ for $\frac{1}{2} \leq f<1$. We note that for $\frac{1}{3} \leq f<1$, the $\mathrm{DPL}_{n}$-extreme function corresponding to the third extreme point of $P \Theta_{1}$ is equal to drlm_2_slope_limit ( $f, 1,2$ ) and thus is extreme. We now prove that for the other case where $0<f<\frac{1}{3}$ the function is extreme as well.

Theorem 2.1. Let $f \in\left(0, \frac{1}{3}\right)$ be real. The $r l m_{-} d p l 1_{-}$ extreme_3a function $\pi$ defined as follows is extreme:

$$
\pi(x)= \begin{cases}\frac{1}{f} x & \text { if } 0 \leq x \leq f \\ \frac{2}{1+2 f} x & \text { if } f<x<\frac{1+f}{2} \\ \frac{1}{2} & \text { if } x=\frac{1+f}{2} \\ \frac{2}{1+2 f} x-\frac{1}{1+2 f} & \text { if } \frac{1+f}{2}<x<1 \\ 0 & \text { if } x=1\end{cases}
$$

Proof. Minimality of $\pi$ follows from [14, Definition 19, Proposition 18 and Corollary 23].

The proof of extremality follows the basic roadmap mentioned in [4, subsection 2.3. Suppose that $\pi=\frac{1}{2}\left(\pi^{1}+\right.$ $\pi^{2}$ ), where $\pi^{1}, \pi^{2}$ are valid functions. Define the additivity domain of $\pi$ as

$$
E(\pi):=\{(x, y) \in \mathbb{R} \times \mathbb{R}: \pi(x)+\pi(y)-\pi(x+y)=0\} .
$$

By [4, Lemma 2.11, 


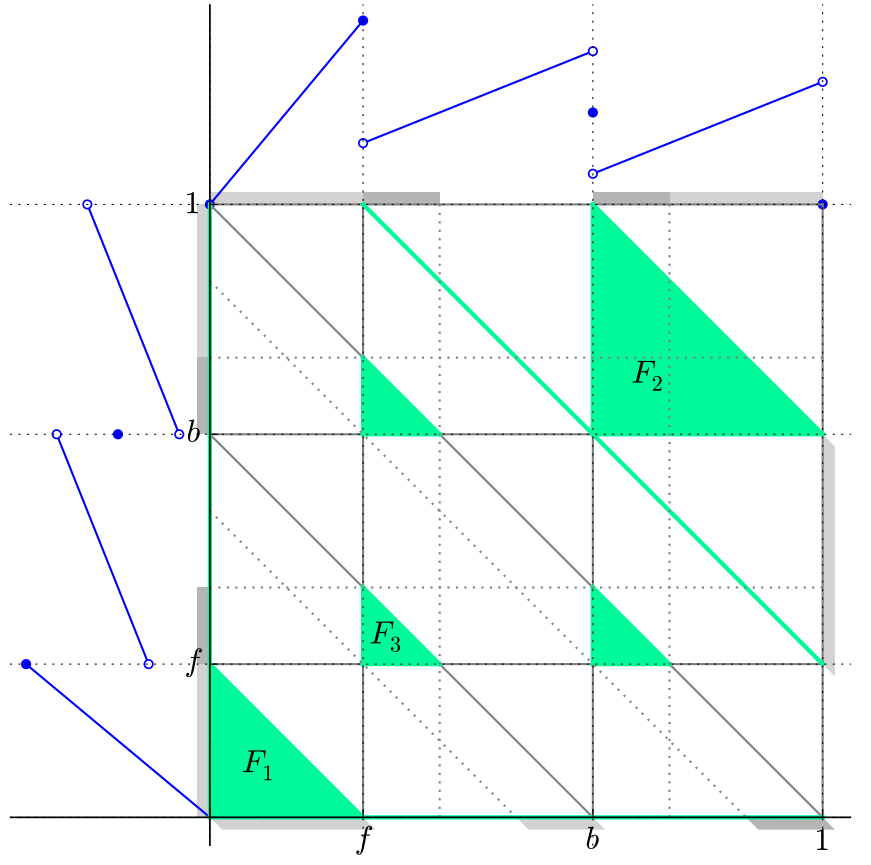

Figure 1: Diagram of the function $r l m_{-}$dpl1_extreme_3a (blue graphs on the top and the left) and its polyhedral complex $\Delta \mathcal{P}$ (gray solid lines). The set $E(\pi)$ is the union of the faces shaded in green. The heavy diagonal green line $x+y=1+f$ corresponds to the symmetry condition (the line $x+y=f$ appears as an edge of $F_{1}$ ). Vertices of $\Delta \mathcal{P}$ do not necessarily project (dotted gray lines) to breakpoints. At the borders, the projections $p_{i}(F)$ of two-dimensional additive faces are shown as gray shadows: $p_{1}(F)$ at the top border, $p_{2}(F)$ at the left border, $p_{3}(F)$ at the bottom and the right borders.

(i) $\pi^{1}, \pi^{2}$ are minimal;

(ii) all subadditivity relations $\pi(x+y) \leq \pi(x)+\pi(y)$ that are tight for $\pi$ are also tight for $\pi^{1}, \pi^{2}$, i.e., $E(\pi) \subseteq$ $E\left(\pi^{1}\right), E\left(\pi^{2}\right)$

(iii) because $\pi$ is continuous from the right at $x=0$, the functions $\pi^{1}, \pi^{2}$ are continuous at all points at which $\pi$ is continuous.

Consider $\pi, \pi^{1}, \pi^{2}$ as solutions to the following system of linear equations:

$$
\left\{\begin{array}{l}
\varphi(0)=0, \\
\varphi(f)=1, \\
\varphi(1)=0, \\
\varphi(u)+\varphi(v)=\varphi(u+v), \quad(u, v) \in E(\pi),
\end{array}\right.
$$

where $\varphi$ is a minimal function that is continuous at all points at which $\pi$ is continuous. We will show that (3) has a unique solution. This will imply that $\pi=\pi^{1}=\pi^{2}$, thereby establishing the extremality of $\pi$.

Following [4, subsection 3.2, we define for any intervals $I, J, K \subseteq \mathbb{R}$ the set

$F(I, J, K):=\{(x, y) \in I \times J: x+y \in K\} \subseteq \mathbb{R} \times \mathbb{R}$.
Define the projections $p_{1}, p_{2}, p_{3}: \mathbb{R} \times \mathbb{R} \rightarrow \mathbb{R}$ by

$$
p_{1}(x, y)=x, \quad p_{2}(x, y)=y, \quad p_{3}(x, y)=x+y .
$$

Consider the closed triangle

$$
F_{1}=F([0, f],[0, f],[0, f])
$$

and the open triangles

$$
\begin{aligned}
& F_{2}=F((b, 1),(b, 1),(1+f, 1+b)), \\
& F_{3}=F((f, b),(f, b),(2 f, b)),
\end{aligned}
$$

where $b=\frac{1+f}{2}$. See Figure 1 for an illustration. Then, for any $(x, y) \in F_{1}$,

$$
\pi(x)+\pi(y)-\pi(x+y)=\frac{x}{f}+\frac{y}{f}-\frac{x+y}{f}=0 .
$$

For any $(x, y) \in F_{2}$,

$\pi(x)+\pi(y)-\pi(x+y)=\frac{2 x-1}{1+2 f}+\frac{2 y-1}{1+2 f}-\frac{2(x+y-1)}{1+2 f}=0$.

For any $(x, y) \in F_{3}$,

$\pi(x)+\pi(y)-\pi(x+y)=\frac{2 x}{1+2 f}+\frac{2 y}{1+2 f}-\frac{2(x+y)}{1+2 f}=0$.

Hence $F_{1}, F_{2}, F_{3} \subset E(\pi)$. By the convex additivity domain lemma 4, Theorem 4.3 applied to the full-dimensional convex set $F_{1}, \varphi$ is affine on the open interval int $\left(p_{1}\left(F_{1}\right)\right)=$ $(0, f)$. (We say that the interval $(0, f)$ is covered.) Then $\varphi$ is uniquely determined on $[0, f]$ since $\varphi(0)=0, \varphi(f)=1$, and $\varphi$ is continuous from the right at 0 and from the left at $f$.

Similarly, by [4, Theorem 4.3 applied to $F_{2}, \varphi$ is affine on $(b, 1)$. Let $s$ be the slope. By symmetry, $\varphi$ is also affine on $(f, b)$ with the same slope $s$. Let $\varphi\left(u^{-}\right)$and $\varphi\left(u^{+}\right)$ denote the left limit and right limit value at $u$, respectively. By the symmetry condition of minimal valid functions 4 , Theorem 2.6, $\varphi\left(f^{+}\right)+\varphi\left(1^{-}\right)=1$ and $\varphi\left(b^{-}\right)+\varphi\left(b^{+}\right)=1$. Let $t=\varphi\left(f^{+}\right)$, and thus $\varphi\left(b^{-}\right)=t+\frac{1-f}{2} s, \varphi\left(b^{+}\right)=$ $1-t-\frac{1-f}{2} s$, and $\varphi\left(1^{-}\right)=1-t$. Also note that $\varphi(1)=0$ and $\varphi(b)=\frac{1}{2}$. Therefore $\varphi$ is uniquely determined by $s$ and $t$.

Now set up two more equations using the additivity relations:

$$
\varphi\left(f^{+}\right)+\varphi\left(f^{+}\right)=\varphi\left(2 f^{+}\right),
$$

which corresponds to the lower left corner of the open triangle $F_{3}$, and

$$
\varphi\left(b^{+}\right)+\varphi\left(b^{+}\right)=\varphi\left(f^{+}\right),
$$

which corresponds to the lower left corner of the open triangle $F_{2}$. Since $0<f<1 / 3$, we have $f<2 f<b$. It follows that $\varphi\left(2 f^{+}\right)=t+f s$. Hence $2 t=t+f s$ by (6) and $2\left(1-t-\frac{1-f}{2} s\right)=t$ by (7). The system has a unique solution, namely $s=\frac{2}{1+2 f}$ and $t=\frac{2 f}{1+2 f}$. This shows that $\varphi$ is unique on $[0,1]$, completing the proof of extremality of $\pi$. 


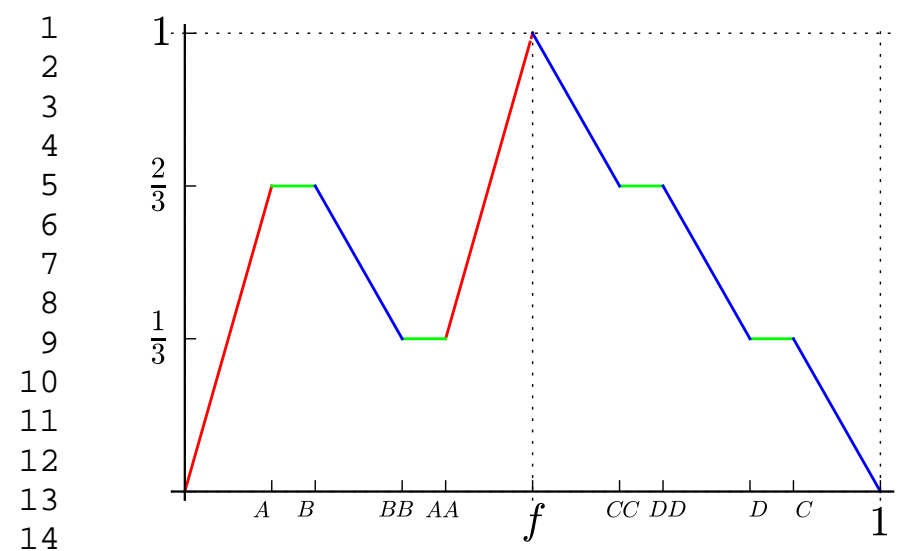

15

16

17

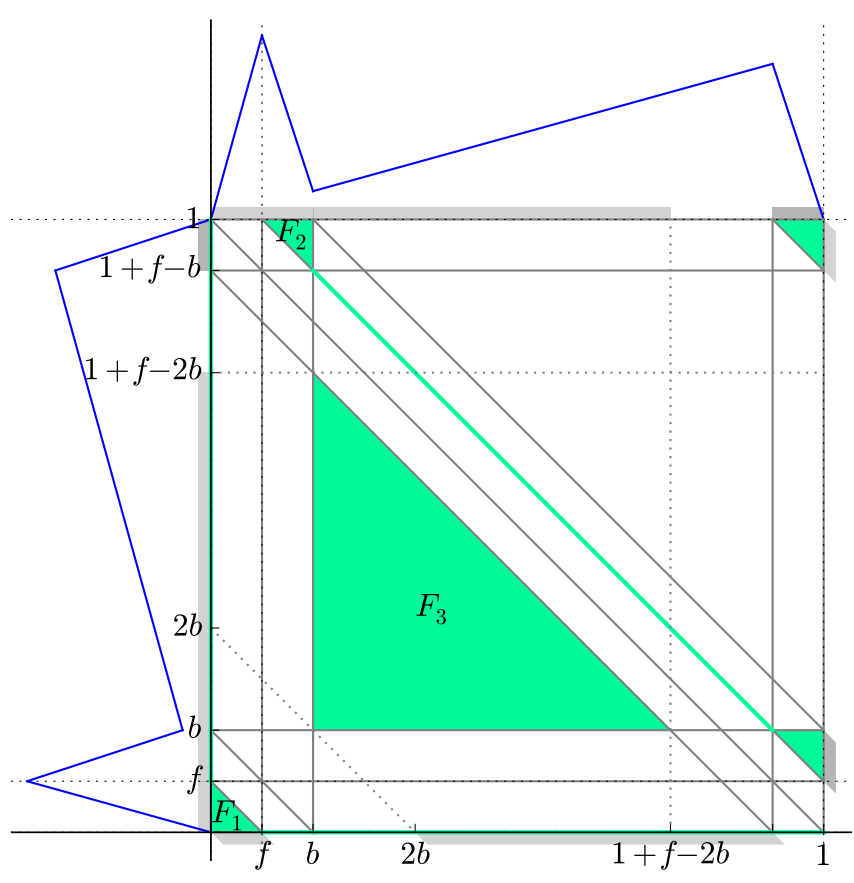

Figure 3: The drlm_backward_3_slope function

cause there is a "height decrease" of 1 in the function from $\pi(f)$ and $\pi(1)$. In fact, this is false whenever the common slope value over the intervals $[A, B],[B B, A A],[C C, D D]$ and $[D, C]$, called $s_{3}$, is non-zero. Since one equation is missing from the system of six equations in six unknowns claimed by the author, the system does not have a unique solution. As a result, $\pi$ is not extreme.

The software 11], as part of which the Electronic Compendium is released, allows us to test the extremality for a given function (i.e., for fixed parameters $f, A, B, C$, and $D)$. Computationally we verified, by randomly generating thousands of functions in the described family, that none of these functions appear to be extreme.

\section{A standard proof of the extremality of drlm backward_3_slope that removes the assumption of rational data}

The function drlm_backward_3_slope Figure 3 was discovered in [7] and proven to be extreme for rational numbers $f$ and $b$ satisfying $f<b<(1+f) / 4<1$. The proof is based on interpolation of extreme functions for finite cyclic groups [1, where the assumption of rational data is needed.

In fact, following the same roadmap as in the proof of Theorem 2.1. one can show that drlm_backward_3_slope is extreme without assuming $f$ and $b$ to be rational numbers.

Theorem 4.1. Let $f$ and $b$ be real numbers such that $0<$ $f<b \leq \frac{1+f}{4}$. The drlm_backward_3_slope function $\pi$ 
defined as follows is extreme:

$$
\pi(x)= \begin{cases}\frac{x}{f} & \text { if } 0 \leq x \leq f \\ 1+\frac{(1+f-b)(x-f)}{(1+f)(f-b)} & \text { if } f \leq x \leq b \\ \frac{x}{1+f} & \text { if } b \leq x \leq 1+f-b \\ \frac{(1+f-b)(x-1)}{(1+f)(f-b)} & \text { if } 1+f-b \leq x \leq 1\end{cases}
$$

Proof. First, it is straightforward to check that the function $\pi$ is symmetric and subadditive. Thus, by 4, Theorem 2.6, $\pi$ is minimal. Second, by the convex additivity domain lemma for continuous functions [4, Corollary 4.9 applied to

$$
\begin{aligned}
& F_{1}=F([0, f],[0, f],[0, f]), \\
& F_{2}=F([f, b],[1+f-b, 1],[1+f, 1+b]), \\
& F_{3}=F([b, 1+f-2 b],[b, 1+f-2 b],[2 b, 1+f-b]),
\end{aligned}
$$

the intervals $[0, f],[f, b],[b, 1+f-b]$, and $[1+f-b, 1]$ are all covered with slope values $s_{1}, s_{2}, s_{3}$, and $s_{2}$, respectively (see Figure 3). Finally, set up a system of equations using $\varphi(0)=\varphi(1)=0, \varphi(f)=1$ and $\varphi(b)+\varphi(b)=\varphi(2 b)$. This system has a unique solution. Therefore, $\pi$ is an extreme function.

\section{A continuous "1-slope function" as a limiting case of Basu et al.'s non-piecewise linear ex- treme function}

Basu, Conforti, Cornuéjols and Zambelli [2] constructed an extreme non-piecewise linear function, disproving a conjecture of Gomory and Johnson [10, section 6.1]. Their function is defined as a limit of continuous piecewise linear functions which are defined as follows. Let $f \in(0,1)$ be real. Consider a sequence of real numbers $\epsilon_{1}>\epsilon_{2}>\ldots$ such that $\epsilon_{1} \leq 1-f$ and

$$
\mu^{-}=(1-f)+\sum_{i=1}^{+\infty} 2^{i-1} \epsilon_{i}<1 .
$$

Basu et al. give a recursive construction of a function $\psi_{n}: \mathbb{R} \rightarrow \mathbb{R}$, each depending on parameters $f$ and $\epsilon_{1}, \ldots, \epsilon_{n}$ the function $\psi_{0}$ is the gmic function [2, section 3]. We state a first observation without proof; see Figure 4 for an illustration.

Observation 5.1. Basu et al.'s functions $\psi_{n}$ are special cases of $k f_{-} n_{-} s t e p_{-} m i r$ functions [12] with the following parameters:

$$
\begin{gathered}
f=f, \quad a_{0}=1, \quad a_{1}=\frac{f+\epsilon_{1}}{2}, \\
a_{i}=\frac{a_{i-1}-\epsilon_{i-1}+\epsilon_{i}}{2}, \text { for } i=2,3, \ldots, n .
\end{gathered}
$$

Basu et al. 2. prove that the sequence formed by these functions converges uniformly and hence gives a continuous limit function $\psi$. The limit function is a fractal, an absolutely continuous, measurable, non-piecewise linear "2-slope function" with the following properties:

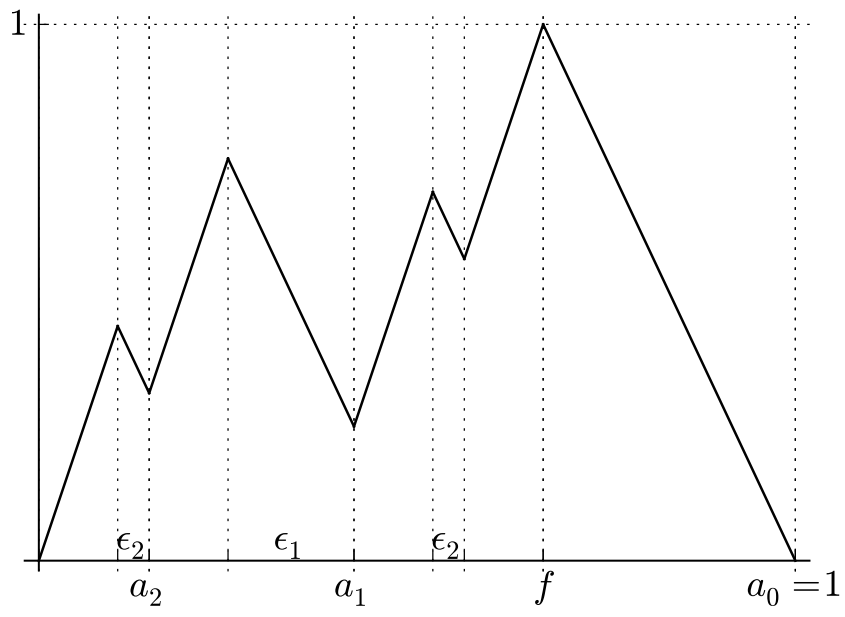

Figure 4: The kf_n_step_mir function

(i) There is a set $X^{-} \subseteq[0,1]$, a countable union of open intervals, of Lebesgue measure $\mu^{-}<1$, on which the function is differentiable with the derivative taking the same, negative value.

(ii) There is a set $X^{+} \subseteq[0,1]$ of measure $\mu^{+}=1-\mu^{-}>$ 0 , which is nowhere dense, i.e., it does not contain any interval. Such a set is sometimes called a fat Cantor set. The function is differentiable on $X^{+}$as well, and the derivative takes the same, positive value on the points of $X^{+}$.

(iii) There is an at most countable subset where the function is not differentiable.

In this note, we consider an interesting fringe case of the same construction, where $\mu^{-}$in (8) equals 1 . We restrict ourselves to the case of geometric sequences, which are the prime examples for the case $\mu^{-}<1$ as well.

So consider the geometric sequence $\left\{\epsilon_{i}: i=1,2, \ldots\right\}$ with common ratio $\frac{1}{q}$ and the first term $\epsilon_{1}=\frac{q-2}{q} f$, where $2<q \leq \frac{2 f}{2 f-1}$ for $f>\frac{1}{2}$ and $2<q$ for $f \leq \frac{1}{2}$. Then $\left\{\epsilon_{i}\right\}$ is a decreasing sequence of positive reals, with $\epsilon_{1} \leq 1-f$ and

$$
\mu^{-}=(1-f)+\sum_{i=1}^{+\infty} 2^{i-1} \epsilon_{i}=1 .
$$

Again let $\psi_{i}$ be the valid function defined in [2, section 3], for $i=1,2,3, \ldots$. Let

$$
\gamma_{i}=f-\sum_{k=1}^{i} 2^{k-1} \epsilon_{k}=\left(\frac{2}{q}\right)^{i} f .
$$

By (9), $\gamma_{i}>0$ for every $i \geq 0$. Thus, by [2, Fact 4.1], $\psi_{i}$ is well-defined.

We will now show by a simple calculation that in our case still uniform convergence holds. The following result replaces [2, Lemma 5.2]. 
Lemma 5.2. The sequence $\psi_{1}, \psi_{2}, \ldots$ is uniformly convergent.

Proof. By [2, Fact 4.1], there are $2^{i}$ intervals where $\psi_{i}$ has positive slope $s_{i}=\frac{1-\gamma_{i}}{(1-f) \gamma_{i}}$, each of length $\frac{\gamma_{i}}{2^{i}}$. Note that $\left|\psi_{i}(x)-\psi_{i+1}(x)\right| \leq s_{i+1} \frac{\gamma_{i}}{2^{i}}$ since the values of the two functions match at the ends of the positive-slope intervals of $\psi_{i}$. Since

$s_{i+1} \frac{\gamma_{i}}{2^{i}}=\frac{1-\gamma_{i+1}}{(1-f) \gamma_{i+1}} \cdot \frac{\gamma_{i}}{2^{i}} \leq \frac{1}{1-f} \frac{\gamma_{i}}{\gamma_{i+1}} \frac{1}{2^{i}}=\frac{q}{2(1-f)} \frac{1}{2^{i}}$,

we know that $\left|\psi_{i}(x)-\psi_{i+1}(x)\right| \leq C \frac{1}{2^{i}}$, where $C=\frac{q}{2(1-f)}$. Therefore, if $n<m$ then

$$
\left|\psi_{n}(x)-\psi_{m}(x)\right| \leq \sum_{i=n}^{m-1} C \frac{1}{2^{i}} \leq \sum_{i=n}^{\infty} C \frac{1}{2^{i}}=C \frac{1}{2^{n-1}} .
$$

This implies that the sequence is Cauchy and hence convergent. Thus the pointwise limit

$$
\psi(x)=\lim _{i \rightarrow \infty} \psi_{i}(x)
$$

of this sequence of functions is well defined. Moreover, since the bound on $\left|\psi_{n}(x)-\psi_{m}(x)\right|$ does not depend on $x$, the above argument immediately implies that the sequence of functions $\psi_{i}$ converges uniformly to $\psi$.

Remark 5.3. When $i$ tends to $\infty, \gamma_{i}$ tends to 0 and the positive slope $s_{i}$ tends to $\infty$. Thus the convergence fails in the sense of $W_{\text {loc }}^{1,1}(\mathbb{R})$, i.e., the limit function is no longer absolutely continuous.

The limit function is a minimal valid function because limits preserve minimality 4, section 6. Since, in general, extremality is not preserved by limits (see again [4, section 6 for a discussion), the extremality of the limit function requires a proof that uses the detailed structure of the functions $\psi_{n}$. The proof in [2] extends verbatim to our case and will not be repeated here.

Hence $\psi$ is a continuous (but not absolutely continuous), measurable, non-piecewise linear, extreme "1-slope function." It is available in the Electronic Compendium as a special case of bccz_counterexample.

Remark 5.4. The function that is constructed by $\mathrm{bccz}_{-}$ counterexample can be exactly evaluated only on the union of the closures of the open intervals that form the set $X^{-}$; for other values, the function will return an approximation. This enables simple computational experiments with this function, but the automated minimality and extremality test is not available.

Open question 5.5. It is an open question if computational methods can be developed that can assist with the discovery, construction, and extremality test of non-piecewise linear functions that are like bccz_counterexample defined as limits of piecewise linear functions.
Table 1: An overview of the Electronic Compendium of extreme functions, available at https://github.com/mkoeppe/ infinite-group-relaxation-code
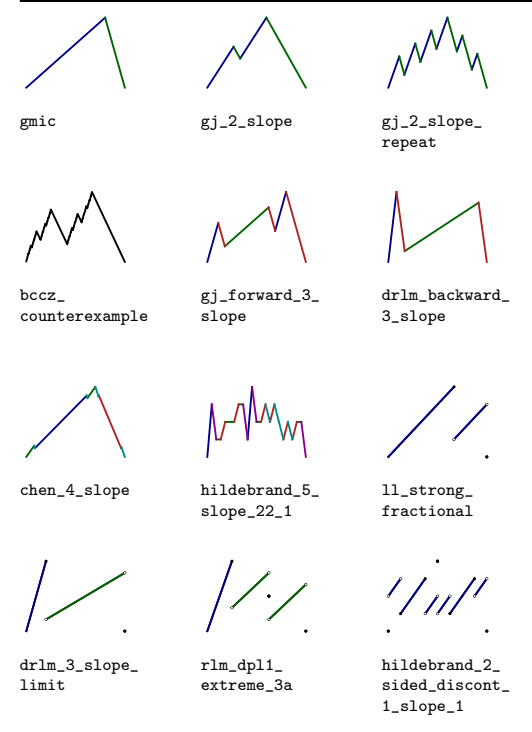
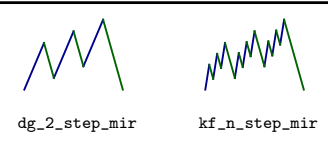

\begin{tabular}{|l|l|l|l} 
gj_2_slope \\
repeat
\end{tabular}
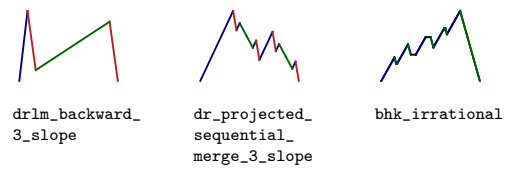
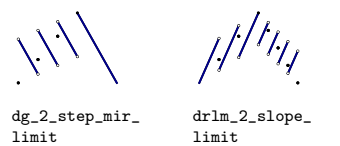
$\frac{\text { dr1m_2- }}{\text { limit }}$
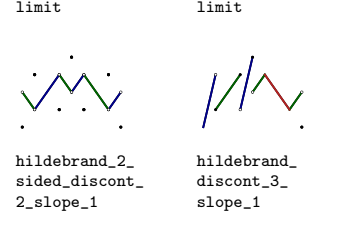

\section{The Electronic Compendium}

The Electronic Compendium is implemented in Python within the framework of the open-source computer algebra system Sage [15. It is available as part of the software [11. It can be run on a local installation of Sage, or online via SageMathCloud.

An overview of the available functions is given in Table 1. These functions are defined in the Sage files extreme functions_in_literature.sage and survey_examples. sage.

Table 2 shows a sample Sage session, illustrating the basic use of the software and the help system. The latter provides a discussion of parameters of the extreme functions, bibliographic information, etc. It is accessed by typing a function name, followed by a question mark.

Following the standard conventions of Sage, the documentation strings contain usage examples with their expected output. If Sage is invoked as

$$
\text { sage -t }\langle\text { filename }\rangle \text {.sage, }
$$

these examples are run and their results are compared to the expected results; if the results differ, this is reported as a unit test failure. This helps to ensure the consistency and correctness of the compendium as we continue to add newly discovered functions or generalize their constructions.

\section{Acknowledgment}

The authors gratefully acknowledge partial support from the National Science Foundation through grant DMS-1320051 awarded to M. Köppe. 
Table 2: A sample Sage session

\section{\# First load the code.}

age: import igp; from igp import *

function and store it in variable $h$.

sage: $\mathrm{h}=\mathrm{gmic}()$

Plot the fun 17:20:34,863 Rational case.

sage : plot_with_colored_slopes $(\mathrm{h})$

\# The documentation string of each function reveals its optional arguments

* usage examples, and bibliographic information.

sage: gmic

Def inition: $\operatorname{gmic}(f=4 / 5)$

Summary:

* Name: GMIC (Gomory mixed integer cut);

* Infinite (or Finite); Dim = 1; Slopes = 2; Continuous; Analysis of subadditive polytope method;

* Discovered [55] p.7-8, Eq.8;

* Proven extreme (for infinite group) [60] p.377, thm.3.3; (finite group) [57] p.514, Appendix 3.

* (Although only extremality has been established in literature, the same proof shows that) gmic is a facet.

Parameters:

$f$ (real) in $(0,1)$

Examples:

[61] p.343, Fig. 1, Example 1

sage: logging.disable (logging. INFO)

\# Suppress output in automatic tests. True

[55]: R.E. Gomory, An algorithm for the mixed integer problem,

[55]: R.E. Gomory, An algorithm for the mixed
Tech. Report RM-2597, RAND Corporation, 1960.

[...]

\# The docstring tells us that we can set the ' $f$ ' value using an optional argument.

sage: $\mathrm{h}=\operatorname{gmic}(1 / 5)$

NF0: $2014-11-18$ 17:21:12,172 Rational case.

INFO:
INTtremality_test $(\mathrm{h}$, show_plots $=$ True
2011-18 $17: 21: 12,177$ pi $(0)=0$

INFO: 2014-11-18 $17: 21: 12,178 \mathrm{pi}$ is subadditive.

INFO: 2014-11-18 $17: 21: 12,178 \mathrm{pi}$ is symmetric.

NFF: 2014-11-18 17:21:12,178 Thus $\mathrm{pi}$ is minimal.

: 2014-11-18 17:21:12,178 Plotting 2d diagram.

.

INFO: 2014-11-18 17:21:12,544 Computing covered intervals...

INF0: 2014-11-18 17:21:12,544 Plotting covered intervals...

INFO: 2014-11-18 17:21:12,776 Plotting covered intervals... done

INF0: 2014-11-18 17:21:12,776 All intervals are covered (or connected-to-covered). 2 components. INF0: 2014-11-18 17:21:12,784 Finite dimensional test: Solution space has dimension 0

(1)

\section{References}

[1] J. Aráoz, L. Evans, R. E. Gomory, and E. L. Johnson, Cyclic group and knapsack facets, Mathematical Programming, Series B 96 (2003), 377-408.

[2] A. Basu, M. Conforti, G. Cornuéjols, and G. Zambelli, A counterexample to a conjecture of Gomory and Johnson, Mathematical Programming Ser. A 133 (2012), no. 1-2, 25-38, doi:10.1007/s10107-010-0407-1.

[3] A. Basu, R. Hildebrand, and M. Köppe, Equivariant perturbation in Gomory and Johnson's infinite group problem. I. The one-dimensional case, Mathematics of Operations Research 40 (2014), no. 1, 105-129, doi:10.1287/moor.2014.0660.

[4] A. Basu, R. Hildebrand, and M. Köppe, Light on the infinite group relaxation, eprint arXiv:1410.8584 [math.OC], 2015.

[5] K. Chen, Topics in group methods for integer programming, Ph.D. thesis, Georgia Institute of Technology, June 2011.

[6] M. Conforti, G. Cornuéjols, and G. Zambelli, Corner polyhedra and intersection cuts, Surveys in Operations Research and Management Science 16 (2011), 105-120.

[7] S. S. Dey, J.-P. P. Richard, Y. Li, and L. A. Miller, On the extreme inequalities of infinite group problems, Mathematical Programming 121 (2010), no. 1, 145-170, doi:10.1007/ s10107-008-0229-6

[8] R. E. Gomory and E. L. Johnson, Some continuous functions related to corner polyhedra, I, Mathematical Programming 3 (1972), 23-85, doi:10.1007/BF01585008

[9] Some continuous functions related to corner polyhedra, II, Mathematical Programming 3 (1972), 359-389, doi: 10.1007/BF01585008

10] , T-space and cutting planes, Mathematical Programming 96 (2003), 341-375, doi:10.1007/s10107-003-0389-3

[11] C. Y. Hong, M. Köppe, and Y. Zhou, Sage program for computation and experimentation with the 1-dimensional GomoryJohnson infinite group problem, 2014, available from https: //github.com/mkoeppe/infinite-group-relaxation-code

[12] K. Kianfar and Y. Fathi, Generalized mixed integer rounding inequalities: facets for infinite group polyhedra, Mathematical Programming 120 (2009), 313-346.

[13] J.-P. P. Richard and S. S. Dey, The group-theoretic approach in mixed integer programming, 50 Years of Integer Programming 1958-2008 (M. Jünger, T. M. Liebling, D. Naddef, G. L. Nemhauser, W. R. Pulleyblank, G. Reinelt, G. Rinaldi, and L. A. Wolsey, eds.), Springer Berlin Heidelberg, 2010, pp. 727-801, doi:10.1007/978-3-540-68279-0_19 ISBN 9783-540-68274-5.

[14] J.-P. P. Richard, Y. Li, and L. A. Miller, Valid inequalities for MIPs and group polyhedra from approximate liftings, Mathematical Programming 118 (2009), no. 2, 253-277, doi: 10.1007/s10107-007-0190-9

[15] W. A. Stein et al., Sage Mathematics Software (Version 6.7), The Sage Development Team, 2015, http://www. sagemath.org.

[16] Y. Zhou, Electronic compendium of extreme functions for the 1-row Gomory-Johnson infinite group problem, 2014, available from https://github.com/mkoeppe/ infinite-group-relaxation-code/ 